\title{
Analytical STEM Investigation of the Post-Synthetic Modification (PMS) of Metal- Organic Frameworks (MOFs): Metal- and Ligand-Exchange in UiO-66
}

\author{
Lucas R. Parent ${ }^{1,2}$, Michael S. Denny², Joseph P. Patterson ${ }^{2,3}$, Patricia Abellan ${ }^{4}$, Quentin M. Ramasse ${ }^{4}$, \\ Francesco Paesani ${ }^{2}$, Seth M. Cohen ${ }^{2}$, and Nathan C. Gianneschi ${ }^{1,2}$ \\ 1. Department of Chemistry, Department of Materials Science and Engineering, and Department of \\ Biomedical Engineering, Northwestern University, Evanston, USA. \\ 2. Department of Chemistry and Biochemistry, University of California, San Diego, San Diego, USA. \\ 3. Laboratory of Materials and Interface Chemistry and Center of Multiscale Electron Microscopy, \\ Department of Chemical Engineering, Eindhoven University of Technology, Eindhoven, NL. \\ 4. SuperSTEM Laboratory, SciTech Daresbury Campus, Daresbury, UK.
}

Post-synthetic modification (PSM) of metal-organic frameworks (MOFs) is a promising route to create novel, and diverse porous nanostructures with tailored functionality for applications from gas storage and separation to catalysis [1]. Recently, post-synthetic exchange (PSE) of metal ions in the secondary-building units (SBUs) of MOFs has been demonstrated in the UiO-66 system, whereby Hf or Ti ions are exchanged for the initial $\mathrm{Zr}$ ions in the SBUs, while appearing to retain structural integrity and porosity [2]. Metal ion PSM (transmetalation) has been observed for both solvent-mediated solid-solid reactions and solid-liquid conditions in "robust, inert" MOFs, such as the UiO-66 system [1,3]. This has been proposed as a route to achieve stable MOF structures with specific metal nodes yet to be directly produced using conventional synthesis (e.g. TiUiO-66). Despite the interest in PSM, very little is understood about the mechanisms of the exchange process that seem to enable the robust conversion of one metal-containing crystalline MOF nanostructure to an equallystable and -crystalline MOF that contains new metal nodes. Furthermore, though exciting property enhancements have been measured and reported for various transmetalated MOFs, which supports the widelyaccepted concept of metal-exchange in these systems, the true extent of the exchange of metal ions on the nanoscale within a single MOF particle has not been accurately analyzed and quantified.

Here, were use (scanning) transmission electron microscopy (STEM) energy dispersive X-ray (EDX) spectroscopy and electron energy loss spectroscopy (EELS) elemental mapping in combination with atomic resolution (S)TEM imaging to probe, map, and quantify the distribution of metal species in MOF nanocrystals in the UiO-66 family of MOFs [4]. Using these nanoscale characterization techniques, we explore PSM "metal exchange" in (Zr)UiO-66 in both Hf-salt and Ti-salt solutions, and in (Hf)UiO-66 in Zr-salt solutions. For all the PMS (metal exchange) conditions we investigate, true replacement of the original metal by the exogenous metal in the MOF nanocrystal does not occur as previously reported. Instead, The EDX elemental mapping data indicates (Figure 1) that essentially none of the original metal has been removed from the nanocrystals, and a surface layer of the exogenous metal is present. Using STEM EELS mapping (Figure 2), we find that the exogenous metal surface-coating is not an epitaxial MOF structure, but rather a metal-oxide layer (lacking carbon and oxygen-rich). We also investigate PSM "ligand exchange" in the (Zr)UiO-66 MOF (I-bdc and Brbdc ligand) using analytical STEM where we find that genuine exchange is operative and appears to be uniform into the bulk of the nanocrystals for both ligand-exchange species we investigated.

Without understanding the fundamental processes that underlie MOF PSM through nanoscale spectroscopic characterization, the MOF field will struggle to interpret any promising results of property enhancements when PSM has been employed, or to develop new PSM routes for tailoring specific functional properties in novel MOFs. In this work, we report the first nanoscale analytical study into PSM processes in individual MOF nanocrystals, finding that PSM processes can, and have been misinterpreted in previous studies where only bulk characterization was employed. Observation/characterization of modified MOFs at length scales 
accessible to STEM has led to a new understanding of the UiO-66 MOF system, and broadly, these data demonstrate the utility of analytical STEM for MOFs and similar nanomaterials [5].

\section{References:}

[1] K. K.Tanabe and S. M. Cohen, Chem. Soc. Rev. 40 (2011), p. 498.

[2] M. Kim et al., J. Am. Chem. Soc. 134 (2012), p. 18082.

[3] C. K. Brozek and M. Dinca, Chem. Soc. Rev. 43 (2014), p. 5456.

[4] M. S. Denny et al., J. Am. Chem. Soc. 140 (2018), p. 1348.

[5] This work was supported by: L.R.P National Institutes of Health (NIH) F32 fellowship award F32EB021859, Army Research Office award W911NF-15-1-0189, NSF grant ECCS-1542148. A portion of this work was performed using the Environmental Molecular Sciences Laboratory (EMSL), located at Pacific Northwest National Laboratory (PNNL), under EMSL user proposal no. 49590. PNNL is operated by Battelle 454 for DOE under contract DE-AC05-76RL01830.

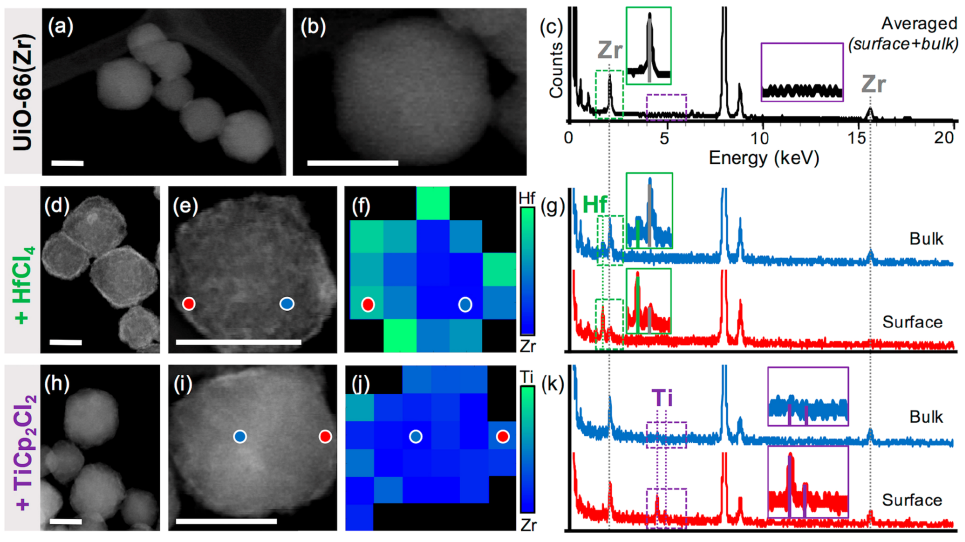

Figure 1. STEM-EDX characterization of $\mathrm{UiO}-66(\mathrm{Zr}), \mathrm{UiO}-66(\mathrm{Zr})+\mathrm{HfCl}_{4}$, and $\mathrm{UiO}-66(\mathrm{Zr})+\mathrm{TiCp}_{2} \mathrm{Cl}_{2}$. All scale bars are $50 \mathrm{~nm}$. (a, b, d, e, h, i) STEM DF images and (c, g, k) STEM-EDX spectra of each of the three samples. (f) STEM-EDX Hf:Zr atomic ratio map for UiO-66(Zr) + $\mathrm{HfCl}_{4}$. (j) STEM-EDX Hf:Ti atomic ratio map for $\mathrm{UiO}-66(\mathrm{Zr})+\mathrm{TiCp}_{2} \mathrm{Cl}_{2}$. (Figure reproduced from ref. [4])
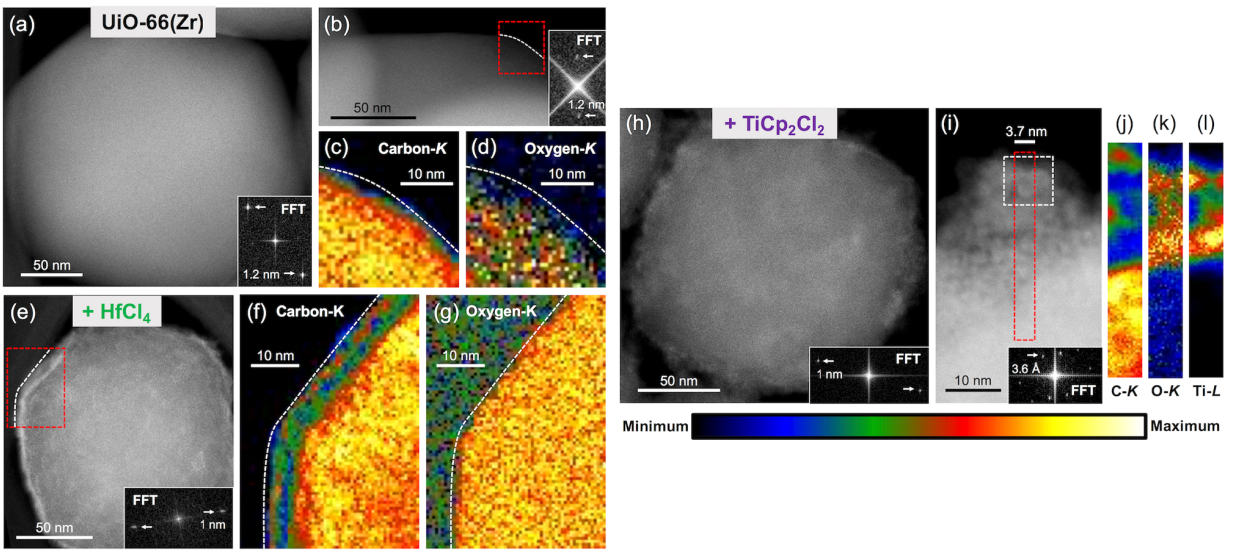

Figure 2. STEM-EELS characterization of UiO-66(Zr), $\mathrm{UiO}-66(\mathrm{Zr})+\mathrm{HfCl}_{4}$, and $\mathrm{UiO}-66(\mathrm{Zr})+\mathrm{TiCp}_{2} \mathrm{Cl}_{2} .(\mathrm{a}, \mathrm{b}$, $\mathrm{e}, \mathrm{h}, \mathrm{i})$ HAADF STEM images and (c, d, f, g, j, k, l) STEM-EELS spectral (elemental) maps (C- $K$, O- $K$, and Ti- $L$ ) of each of the three samples. (Figure reproduced from ref. [4]) 